\title{
Valence Asymmetries in the Human Amygdala: Task Relevance Modulates Amygdala Responses to Positive More than Negative Affective Cues
}

\author{
Paul E. Stillman ${ }^{1}$, Jay J. Van Bavel ${ }^{2}$, and William A. Cunningham ${ }^{1,3}$
}

\begin{abstract}
Organisms must constantly balance appetitive needs with vigilance for potential threats. Recent research suggests that the amygdala may play an important role in both of these goals. Although the amygdala plays a role in processing motivationally relevant stimuli that are positive or negative, negative information often appears to carry greater weight. From a functional perspective, this may reflect the fact that threatening stimuli generally require action, whereas appetitive stimuli can often be safely ignored. In this study, we examine whether amygdala activation to positive stimuli may be more sensitive to task goals than negative stimuli, which are often related to self-preservation concerns. During fMRI, participants were
\end{abstract}

\section{INTRODUCTION}

A zebra keeps an eye out for predators as it bends over to take a drink at a watering hole in the Serengeti National Park in Tanzania. Even the simple act of quenching its thirst is fraught with the risk of being pulled into the water by a crocodile or attacked from behind by a lion. This simple dynamic captures a fundamental tension in goal pursuit across species-the need to engage in approach-related goal pursuit while maintaining constant vigilance for potentially fatal threats. Through the process of evolution, many of the processes that guide attention to important stimuli are thought to be relatively automatic (Öhman \& Mineka, 2001). This is especially true for negative or threatening stimuli, given that failing to respond to negative stimuli could be fatal. Across multiple domains and methodologies, negative stimuli, events, and situations consistently elicit greater psychological reactions (Kahneman \& Tversky, 1979), hold greater sway in evaluations (David, Green, Martin, \& Suls, 1997), resist adaptation (Sheldon, Ryan, \& Reis, 1996; Brickman, Coates, \& Janoff-Bulman, 1978), and capture attention faster (Pratto \& John, 1991) than their positive counterparts. This "negativity bias" toward aversive cues

\footnotetext{
${ }^{1}$ The Ohio State University, ${ }^{2}$ New York University, ${ }^{3}$ University of Toronto
}

presented with two images that varied on valence and extremity and were instructed to focus on one of the images. Results indicated that negative stimuli elicited greater amygdala activity regardless of task relevance. In contrast, positive stimuli only led to a relative increase in amygdala activity when they were task relevant. This suggests that the amygdala may be more responsive to negative stimuli regardless of their relevance to immediate goals, whereas positive stimuli may only elicit amygdala activity when they are relevant to the perceivers' goals. This pattern of valence asymmetry in the human amygdala may help balance approach-related goal pursuit with chronic self-preservation goals.

appears to operate relatively automatically and unconsciously (Ito, Larsen, Smith, \& Cacioppo, 1998).

In their classic paper on valence asymmetries, Baumeister, Bratslavsky, Finkenauer, and Vohs (2001, see also Rozin \& Royzman, 2001) argued that "bad is stronger than good"that negative events hold greater sway over our subjective experience than positive events. From a functional perspective, bad may be stronger than good because the costs of failing to attend to negative objects and events are, on average, much higher than the costs of missed positive opportunities (Öhman, Flykt, \& Esteves, 2001; Öhman \& Mineka, 2001). In this way, negative objects and events may be chronically relevant because they are inherently tied to our powerful self-preservation motives-negative stimuli can signal an imminent and fatal threat to our survival. The relevance of positive, appetitive stimuli, on the other hand, is often based more on one's current goals. Whereas positive stimuli may only capture attention when certain goals render them relevant (e.g., when a thirsty zebra encounters a watering hole), the affective system may be chronically tuned to detect negative stimuli (e.g., the eyes of a crocodile lurking just below the surface of the water). In this way, negative stimuli are more likely to be biologically significant in that they often signal important survival concerns. In this study, we examine how the human brain might balance the need for appetitive goaldirected attention with constant vigilance for potential 
threats. Specifically, we tested whether the amygdala responds to negative stimuli regardless of their relevance to current goals, whereas its response to positive stimuli may be more contextually driven.

The amygdala - a small brain region located deep in the medial-temporal lobe-is known to be critically involved in the processing of affective information (Kim et al., 2011; Whalen \& Phelps, 2009; Sander, Grafman, \& Zalla, 2003; Canli, Zhao, Brewer, Gabrieli, \& Cahill, 2000; Adolphs, Tranel, \& Damasio, 1998; Kluver \& Bucy, 1939). Based on classic neuroimaging research, the amygdala has been linked to fear (LeDoux, 2000; Isenberg et al., 1999; Morris et al., 1996) or negative stimuli (Cunningham, Johnson, Gatenby, Gore, \& Banaji, 2003; Hariri, Tessitore, Mattay, Fera, \& Weinberger, 2002) and appears to play an important role in guiding attention toward affectively significant information (Vuilleumier, 2005; Anderson \& Phelps, 2001). This research has led some to characterize the amygdala as a "fear center" used to coordinate adaptive responses to potential threats in the environment (e.g., Isenberg et al., 1999; Morris et al., 1996) and this region has been hypothesized to be the neural underpinning for the negativity bias (e.g., Öhman \& Mineka, 2001).

To survive, however, organisms must move beyond vigilance for threats and employ attention to facilitate the attainment of appetitive stimuli. Given this need, most organisms are able to orient toward motivationally relevant stimuli, which are determined by a combination of need state and the affordances within the environment. Indeed, people have enhanced accessibility of goalrelevant stimuli (Förster, Liberman, \& Friedman, 2007), which may enhance perceptual awareness (Anderson, 2005; Vuilleumier, 2005; Anderson \& Phelps, 2001; Bruner \& Goodman, 1947). For instance, food-related words are easier to recognize when one is hungry than when one is satiated (Radel \& Clément-Guillotin, 2012). Likewise, what is positive or negative for one's appetitive goals often changes as a function of the current context. For instance, researchers have demonstrated that, given the right affective context, the negativity bias can be eliminated by increasing participants' sensitivity to positive stimuli (Smith et al., 2006).

Similarly, work in affective neuroscience supports the notion that the amygdala is involved in processing affectively relevant stimuli or information, including both positive and negative stimuli (see Cunningham \& Brosch, 2012), and in particular when related to biologically relevant outcomes (Davis \& Whalen, 2001; Whalen, 1998; see also Holland \& Gallagher, 1999). For example, in the case of the zebra, both eating and getting eaten constitute biologically relevant outcomes, and the amygdala may help promote adaptive action in the presence of stimuli that relate to those outcomes. Supporting this more general model of the amygdala, several studies have found that the amygdala responds to the extremity of affective stimuli rather than valence per se (Cunningham, Raye, \&
Johnson, 2004; Anderson, Christoff, Stappen, et al., 2003; Small et al., 2003; Breiter et al., 1996).

A functional affective system must be able to align attention and behavior in light of one's current goals. Indeed, research has found that the amygdala can be modulated or tuned by current motivational concerns (Cunningham, Van Bavel, \& Johnsen, 2008; Sander et al., 2003). For instance, Cunningham and colleagues (2008) presented participants with the names of famous people (e.g., Hillary Clinton) and asked them to evaluate only the positive features, only the negative features, or both. They found that when participants rated celebrities' negative features, amygdala activity was associated with the degree of negative information associated with the celebrity. Likewise, when rating positive features, amygdala activity was associated with the degree of positive information associated with the celebrity. However, when making overall attitude judgments that incorporated positivity and negativity, amygdala activity was greatest to celebrities who were extremely positive or negative. These findings suggest that the amygdala is responsive to motivational relevance of stimuli rather than their sheer negativity (Cunningham \& Brosch, 2012; Cunningham et al., 2008; Sander et al., 2003).

Given the complex nature of the world, it is important to have an affective system that evaluates stimuli according to our current goals. However, recent meta-analyses (e.g., Ferguson \& Bargh, 2004) have demonstrated that amygdala activity is the most reliable predictor relative to other brain regions when differentiating between positive and negative stimuli (Lindquist, Wager, Kober, BlissMoreau, \& Barrett, 2012). This suggests that the amygdala may, in fact, display a "negativity bias." Indeed, even Cunningham and colleagues (2008) found that participants had amygdala activity to negative information even when this information was not task relevant (i.e., when people were rating only positive features). Although the negativity bias can be reduced under certain conditions, a close look at the evidence suggests this has largely been achieved through increasing sensitivity to positive stimuli as opposed to decreasing sensitivity to negative stimuli (e.g., Smith et al., 2006). Thus, thirst may heighten sensitivity toward water without reducing vigilance for crocodiles.

We suspect that both views may contain elements of truth: The affective system may maintain constant vigilance to certain classes of stimuli that have chronic motivational relevance (e.g., negative, aversive stimuli) but be tuned to other classes of stimuli when they are motivationally relevant (e.g., positive, appetitive stimuli). In other words, the amygdala may help facilitate the functional balance between approach and avoidance motivations - enhancing processing of stimuli that are relevant to current goal pursuit while maintaining a constant vigilance for potentially harmful stimuli. Thus, we reasoned that the amygdala would respond to extremely positive or negative stimuli that are relevant to one's current task or goals and respond to negatively valenced 
stimuli regardless of whether these stimuli are relevant to one's focal task goals.

Initial support for these hypotheses comes from research investigating the role of attention in amygdala activation to emotional stimuli. A large body of research has documented the complex relationship between attention and amygdala activity, finding that attention is necessary to elicit amygdala activation to emotional faces in some, but not all contexts (Vuilleumier \& Huang, 2009; Pessoa, 2005, 2008; Williams, McGlone, Abbott, \& Mattingley, 2005; Vuilleumier, Armony, Driver, \& Dolan, 2001). Several researchers have found, for instance, that although responses to fearful faces are generally unaffected by attentional manipulations (e.g., Anderson, Christoff, Panitz, De Rosa, \& Gabrieli, 2003; Vuilleumier et al., 2001) contextual and personality features can modulate this relationship (e.g., high cognitive demands on attention; Bishop, Jenkins, \& Lawrence, 2007; Pessoa, McKenna, Gutierrez, \& Ungerleider, 2002). In one study, for instance, Williams and colleagues (2005) found that attention to happy, but not fearful, faces was modulated by attention (compared with a neutral control). In the present research, we leverage this past work by having our manipulation of task relevance rely in part on drawing attention to one of two images.

\section{This Study}

We conducted an fMRI experiment in which we measured BOLD activity in the amygdala while participants were presented with positive, negative, and neutral images from the International Affective Picture System (IAPS; Lang, Bradley, \& Cuthbert, 2008). For each trial, participants viewed two images side by side simultaneously. Following past research, we then manipulated one image to be the "task-relevant" image, with the other as "task-irrelevant," by instructing participants to selectively focus on one image during each trial. To enhance the affective response to the images, as well as to accentuate differences in relevance, participants were asked to immerse themselves in the picture-thinking about what thoughts and feelings they would have if placed in the scene. This manipulation was intended to focus participants' attention to a single image and follows previous work manipulating task relevance via attentional focus (e.g., Vuilleumier et al., 2001). This procedure further allowed us to manipulate which image was task relevant independent of the valence (from positive, to neutral, to negative) and extremity of both images. Recent research also suggests that individuals can maintain attentional focus on a target image with minimal interference of the task-irrelevant image (Kappenman, MacNamara, \& Proudfit, 2014). If the amygdala responds to both positive and negative stimuli that are task relevant, but primarily to negative stimuli that are task irrelevant, then the amygdala should show a curvilinear relationship with valence (extremity) of task-relevant stimuli and a linear relationship with valence of task-irrelevant stimuli.

\section{METHODS \\ Participants}

Seventeen participants ( 14 women, mean age $=20$ years) provided informed consent and were paid $\$ 30$ for participating in the experiment. Data from two participants were excluded because of scanner malfunction, resulting in 15 total participants $\left(13\right.$ women $\left.^{1}\right)$. Participants reported no abnormal neurological history and had normal or corrected-to-normal vision.

\section{IAPS Image Selection}

To ensure that the qualities of the task-relevant and taskirrelevant images were, on average, equivalent, we used a single pool of 358 images (normed valence ratings varying from 2.54 to 8.34 with mean $=5.43$ on a 9-point scale from $1=$ low pleasure to $9=$ high pleasure; see Appendix for full list). The images in the pool were selected so as to have a near-continuous range of valences. Because positive and negative images can differ in extremity, we intentionally selected images such that positive and negative images would be roughly equated on extremity. To this end, we omitted from our pool both intensely negative IAPS images as well as the erotic positive images. We randomly assigned images to be task-relevant versus task-irrelevant ${ }^{2}$ (examination of normed valence and extremity ratings suggest this randomization strategy achieved the desired effect-there were no average differences between the task-relevant and task-irrelevant images).

\section{Procedure}

Participants arrived at the Queen's University imaging center and were informed they were participating in a study exploring how the brain is organized to evaluate stimuli. After providing informed consent, participants completed five functional runs, each consisting of 19 trials, for a total of 95 trials per participant. On each trial, participants were presented with two randomly selected sideby-side IAPS images. Each image was roughly 30\% of the width of the screen, with a small gap in between images (see Figure 1). We displayed a series of "\#\#\#\#\#\#" signs above one of the images (left-right counterbalanced), and participants were instructed to:

Attend to just that picture. Try to imagine being in the situation you see in the picture and seeing the visual image. Think about the thoughts that you would have, appraise the situation, and simulate the feelings that you would have. Remember, this is just for the picture that has been selected. 


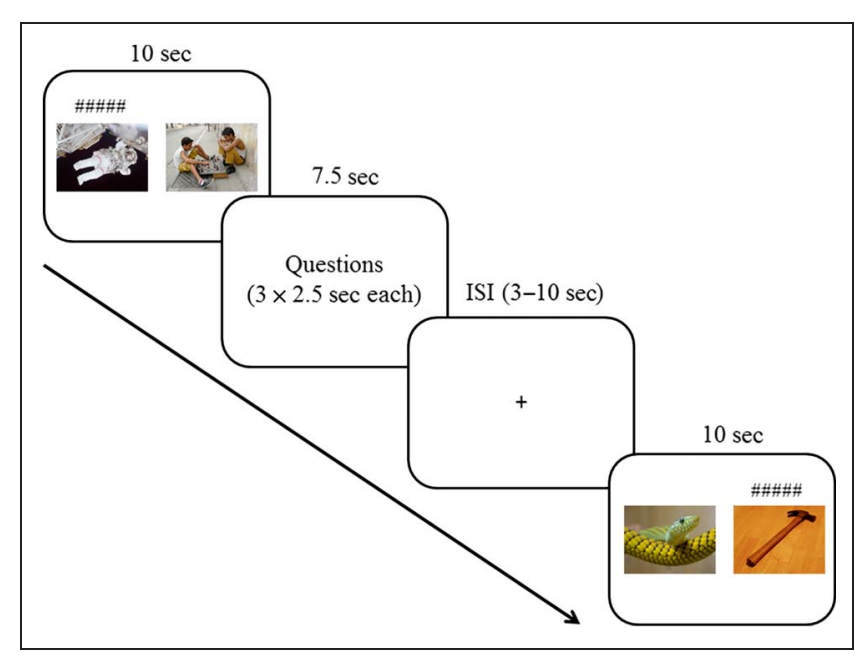

Figure 1. Schematic of the study design. Participants saw two images side by side for $10 \mathrm{sec}$ and were asked to immerse themselves in the image with the "\#\#\#\#\#" above it. Following each trial, participants answered three questions with button boxes (what emotional experience accompanied the image, how positive or negative their response was, and how intense their emotional response was), followed by a variable ISI (average ISI $=4.8 \mathrm{sec}$ ), followed by another trial. Images are not shown to scale.

This method allowed us to independently manipulate both the valence of the stimuli displayed to participants as well as which stimulus was task relevant. Both images were displayed for $10 \mathrm{sec}$. Following each image, participants answered the following questions using two 4-button response boxes: (1) what emotional experience accompanied the images $(1=$ anger, $2=$ fear , $3=$ disgust, $4=\operatorname{sad}, 5=$ joy, $6=$ calm, $7=$ lust, $8=$ proud $),{ }^{3}(2)$ how positive or negative was your response to the attended image (ranging from $1=$ your most negative reaction to $8=$ your most positive reaction), and (3) how emotionally intense was your response to the attended image (from $1=$ no emotional response to 8 = strong emotional response). These questions were immediately followed by a variable intertrial interval (average ITI $=4.8$ sec; see Figure 1 ).

\section{fMRI Parameters}

Neuroimaging was conducted using a Siemens 3T Trio scanner (Erlangen, Germany). Because our hypotheses focused solely on the amygdala, we used a functional sequence that covered subcortical regions at a higher resolution than other regions (with a corresponding loss of coverage in other regions of the brain). Functional images were acquired using a single-shot gradient-echoplanar pulse sequence (echo time $=28 \mathrm{msec}$, repetition time $=2.5 \mathrm{sec}$, in-plane resolution $=2.0 \times 2.0 \mathrm{~mm}$, matrix size $=64 \times 64$, field of view $=128 \mathrm{~mm}$ ).

\section{fMRI Preprocessing and Analysis}

We prepared the data using FSL (University of Oxford, Oxford, UK). Data preprocessing was carried out using FEAT (FMRI Expert Analysis Tool) Version 6.00, part of FSL (FMRIB's Software Library, www.fmrib.ox.ac.uk/fsl). The following preprocessing transformations were applied: motion correction using MCFLIRT (Jenkinson \& Smith, 2001), nonbrain removal using BET (Smith, 2002), spatial smoothing using a Gaussian kernel of FWHM $6 \mathrm{~mm}$, grand mean intensity normalization of the entire 4-D data set by a single multiplicative factor, and high-pass temporal filtering (Gaussian-weighted least-squares straight-line fitting, with sigma $=45 \mathrm{sec}$ ). Following this, registration to high-resolution structural and Montreal Neurological Institute standard space images was performed using FLIRT (Jenkinson, Bannister, Brady, \& Smith, 2002; Jenkinson \& Smith, 2001).

To model the multiple parameters associated with our model, we constructed a multilevel model in which the BOLD response was predicted from the valence and the valence-squared values (extremity) for both the task-relevant and task-irrelevant images. To estimate amygdala activation for each image pair, we obtained a beta-weight estimate for each individual trial for each participant using the individual modulation command in AFNI (-stim_times_IM; see Mumford, Turner, Ashby, \& Poldrack, 2012, for a similar analysis strategy). These betaweights represent the degree of activation for each voxel in response to each trial, as opposed to the average activation across a run or set of runs. For the first set of analyses, we extracted the average activation of all voxels in the amygdala (as defined by the Harvard-Oxford atlas provided with FSL) for each trial (Figure 3A) as well as the individual activation for each voxel in this region.

For both sets of analyses, the amygdala response was modeled on a trial-by-trial basis, with hemisphere of activation as the Level 1 variable, trial as the Level 2 variable, and subject as the Level 3 variable. ${ }^{4}$ Hemispheric location did not interact with any of our predictors, suggesting our effects are not significantly stronger in the left versus right amygdala (all $p s>.2$ ). As such, we only include hemispheric activation as a covariate (our results remain significant when this is not included in the model). This analysis strategy allows us to test the independent effect of multiple continuous predictors while simultaneously increasing our power.

\section{RESULTS}

\section{Valence and Extremity Indices}

Because we were interested in the influence of the valence and extremity on amygdala activation for both task-relevant and task-irrelevant images, we created valence and extremity indices (based on normed IAPS ratings) for the task-relevant and -irrelevant image for 
each trial. To compute valence ratings, we zero-centered the normed valence ratings provided by the IAPS database for the task-relevant image $\left(V_{\mathrm{R}}\right)$ and task-irrelevant image $\left(V_{\mathrm{I}}\right)$. We then took the squared term of these ratings, yielding $V_{\mathrm{R}}^{2}$ and $V_{\mathrm{I}}^{2}$. Because these ratings are zerocentered, their squared term represents their extremity independent of valence (see Cunningham et al., 2008). We focus on this measure of extremity rather than the normed arousal indices supplied in the IAPS database because these indicators of extremity more closely match the concept of stimulus extremity rather than intensity (see also Cunningham et al., 2008). Note, however, that the extremity and arousal indices are highly correlated, $r=.54$, and that our results are virtually identical regardless of the index used. This resulted in four indices for each trial for each participant.

\section{Behavioral Results}

To confirm that participants were indeed attending to the indicated stimulus, participants' posttrial valence ratings were modeled as a function of the normed valence ratings for the task-relevant image $\left(V_{\mathrm{R}}\right)$ in a hierarchical linear model with trial as the Level 1 variable and subject as the Level 2 variable. As predicted, there was a significant positive relationship between participants' ratings and the normed valence ratings $(\gamma=1.23, S E=.04$, $p<.001$, average $r=.64$; see Figure 2). We then performed the same model but instead used the taskrelevant extremity $\left(V_{\mathrm{R}}{ }^{2}\right)$ ratings to predict participants' in-scanner ratings of how extreme their reaction was to the images. Again, as predicted, participants' ratings were significantly positively related to the normed extremity index $(\gamma=.82, S E=.05, p<.001$, average $r=.37)$. In contrast, the valence ratings for the task-irrelevant image $\left(V_{\mathrm{I}}\right)$ were only weakly related to participants' post- trial ratings $(\gamma=.1, S E=.05, p=.06$, average $r=.05)$, and the extremity ratings for the task-irrelevant image $\left(V_{\mathrm{I}}^{2}\right)$ were not significantly related to participants' posttrial extremity ratings $(\gamma=.05, S E=.06, p=.40$, average $r=.02)$. Together, these data suggest that our manipulation of task relevance was successfully influencing participants' evaluations on the subsequent questions. ${ }^{5}$

\section{Neuroimaging Results}

If the amygdala is responsive to both positive and negative task-relevant stimuli, but primarily responsive to negative stimuli that is task-irrelevant, its activity should be correlated with the extremity of task-relevant images and the valence of task-irrelevant images. To test this hypothesis, we ran the three-level hierarchical linear model discussed above to predict amygdala activation using the normed valence and extremity of the taskrelevant and task-irrelevant images $\left(V_{\mathrm{R}}, V_{\mathrm{R}}{ }^{2}, V_{\mathrm{I}}\right.$, and $\left.V_{\mathrm{I}}^{2}\right)$, controlling for the hemispheric location of the activity. Consistent with our hypotheses, bilateral amygdala activity to the task-relevant image was significantly associated with extremity $\left(V_{\mathrm{R}}{ }^{2} ; \gamma=23.96, S E=8.77, p=.007\right.$; see Figure $3 \mathrm{~B})$, but not valence $\left(V_{\mathrm{R}} ; p>.45\right)$. This suggests that the amygdala was responding to extremity rather than valence for the task-relevant image. In contrast, bilateral amygdala activity to the task-irrelevant images was significantly associated with valence $\left(V_{\mathrm{I}} ; \gamma=-13.98\right.$, $S E=6.95, p=.04$; Figure $3 \mathrm{~B})$, but not extremity $\left(V_{\mathrm{I}}^{2}\right.$; $p>$.8). This suggests that the amygdala was associated with negativity rather than extremity of the task-irrelevant image. ${ }^{6}$ There were no higher-order interactions between regressors (all $p s>.15$ ). To add support to these analyses, we repeated the same hierarchical linear model, using the normed arousal indices from the IAPS database instead of our extremity index. These analyses mirrored
Figure 2. Correlation between the normed valence (left) and extremity (right) ratings and participant ratings of valence and extremity while in the scanner. These results suggest that participants' in-scanner ratings were much more driven by the attended rather than the unattended image.

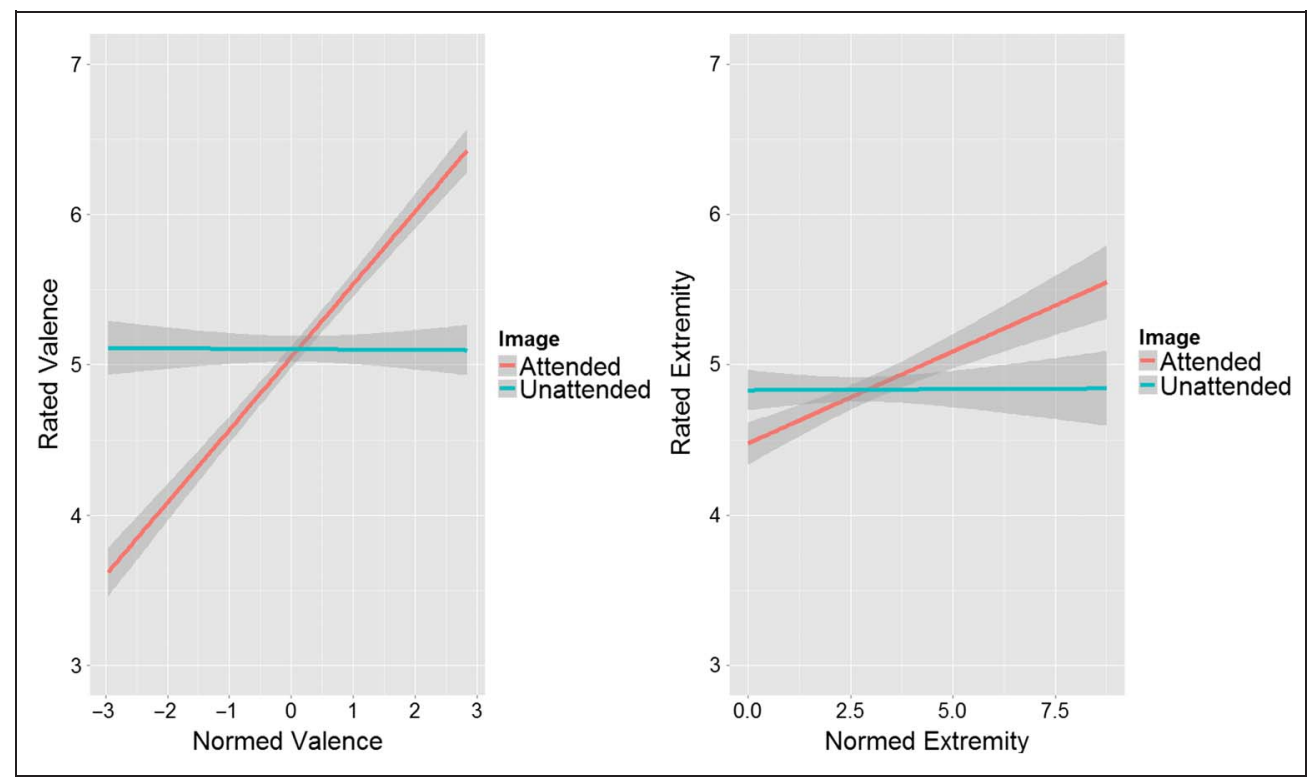



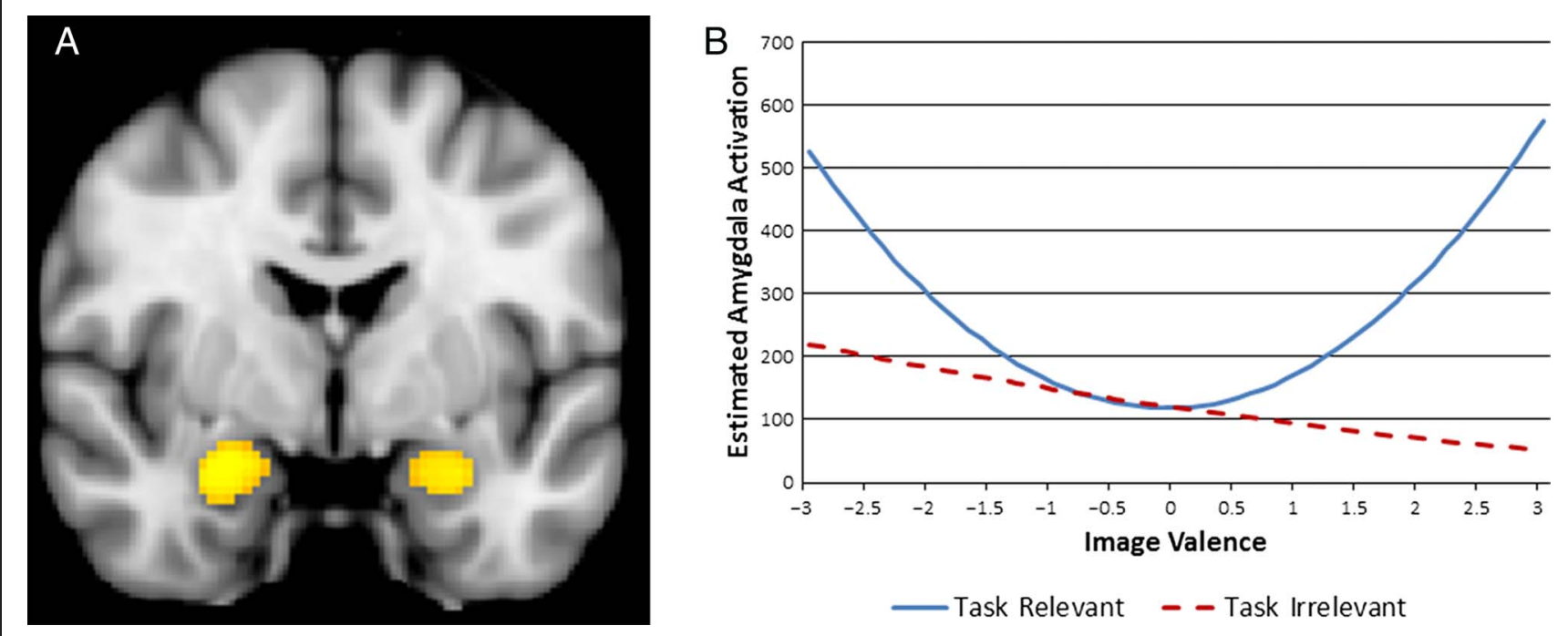

Figure 3. (A) Bilateral amygdala ROI used to extract activation to individual trials $(y=-2)$. (B) Estimated amygdala activation to both task-relevant (solid) and task-irrelevant (dashed) images. Estimates were obtained using $\gamma$ from the hierarchical model discussed in the Methods section and correspond to greater bilateral amygdala activation. The $x$ axis represents the valence of the image (higher is more positive, 0 is neutral, lower is more negative).

our results using the extremity index. Once again, bilateral amygdala activity was significantly associated with all/both positive and negative arousing task-relevant images $\left(V_{\mathrm{R}}^{2}\right.$; $\gamma=32.00, S E=11.78, p=.007)$, but only significantly associated with negatively valenced task-irrelevant images, irrespective of arousal $\left(V_{\mathrm{I}} ; \gamma=-26.70, S E=13.82, p=.05\right)$. Neither task-relevant valence nor task-irrelevant arousal were significantly related to amygdala activation $(p s>.2)$.
To further investigate the structural location of these effects in the amygdala, we conducted a voxel-wide test for overlap of regions. This also allowed us to determine whether our two effects (task-relevant extremity and taskirrelevant valence) were dissociated within the amygdala or whether the same regions of the amygdala responded to both. To this end, we extracted the trial activation betas for each voxel in the amygdala (as opposed to
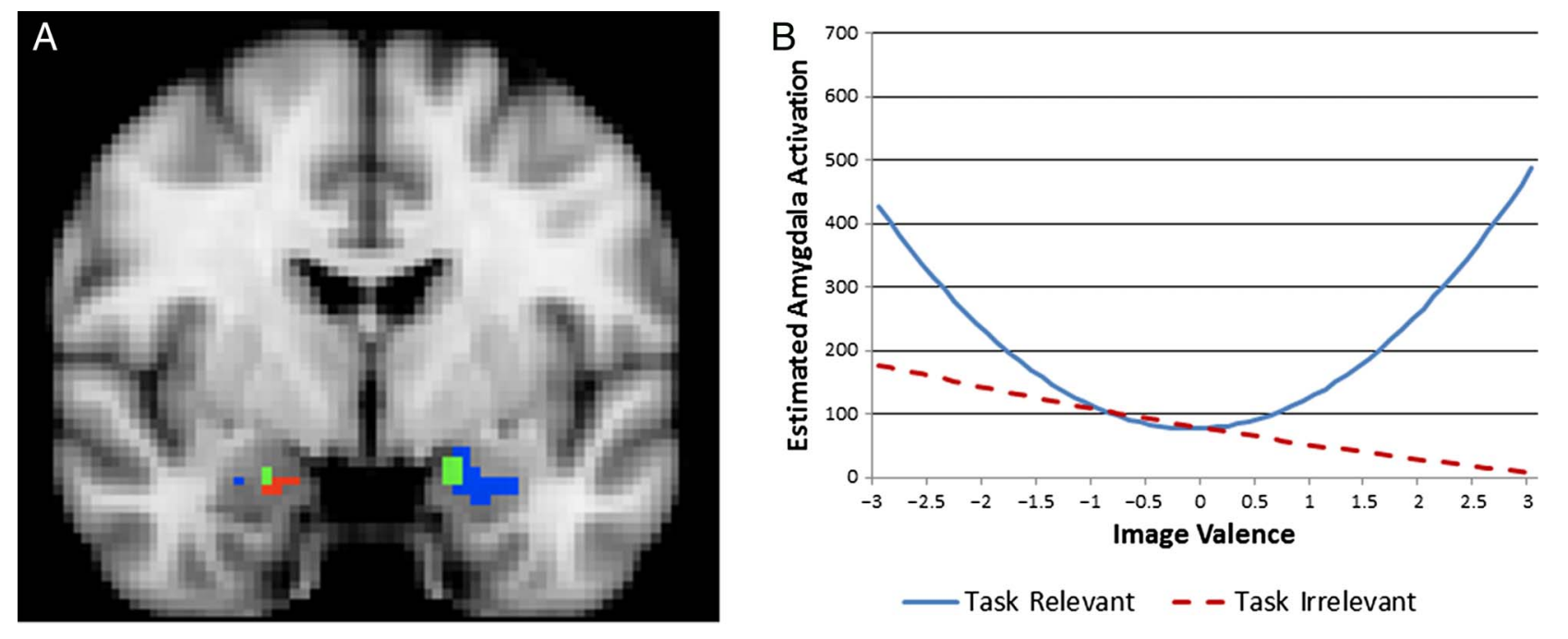

Figure 4. (A) Amygdala activation for task-relevant extremity (blue), task-irrelevant valence (red), or both (green) at $y=-2$. Voxels pictured are those for which the corresponding gamma was significantly positive (task-relevant extremity) or significantly negative (task-irrelevant valence) at $p<.05$ in the multilevel model. (B) Estimated bilateral amygdala activation to both task-relevant (solid) and task-irrelevant (dashed) images for just those voxels that were significantly related to both task-relevant extremity and task-irrelevant valence (green voxels in A). Estimates were obtained using $\gamma$ from the hierarchical model discussed in the Methods section and correspond to greater amygdala activation. The $x$ axis represents the valence of the image (higher is more positive, 0 is neutral, lower is more negative). 
the average as we had done in the analyses above; voxel size $=2 \times 2 \times 2$ ). We then separately ran a multilevel model on each voxel in the amygdala. Consistent with our hypotheses and replicating the above analyses, out of a possible 295 voxels, 115 voxels were significantly positively related to task-relevant extremity $\left(V_{\mathrm{R}}{ }^{2}\right.$; average $\gamma=31.00$, average $p=.02$ ) and 54 voxels were significantly negatively related to task-irrelevant valence $\left(V_{\mathrm{I}}\right.$; average $\gamma=-28.55$, average $p=.02)$. A conjunction analysis indicated that 13 voxels were significantly activated in response to both extremity for the task-relevant image and valence for the task-irrelevant image (see Figure 4), which surpassed what would be expected by chance given a Monte Carlo simulation. There were no significant reversals for either of these effects, nor were there were any significant voxels for either task-relevant valence $\left(V_{\mathrm{R}}\right.$; average $\left.p=.59\right)$ or task-irrelevant extremity $\left(V_{\mathrm{I}}^{2}\right.$; average $\left.p=.66\right)$.

This analysis also allows us to investigate whether our effects are driven by one or more subnuclei of the amygdala. Using the Jülich atlas provided by FSL, we investigated whether our voxels were located in one of the three subnuclei of the amygdala (superficial, laterobasal, and centromedial). This analysis revealed that 11 of the 13 voxels significantly activated for both task-relevant extremity and task-irrelevant valence were located in the superficial subnucleus. Past research has shown that the superficial subnucleus of the amygdala is highly reactive to faces (Goossens et al., 2009) and that the superficial subnucleus has greater connectivity to orbitofrontal regions (Bach, Behrens, Garrido, Weiskopf, \& Dolan, 2011), suggesting that this region may be particularly involved in the processing of motivationally relevant objects. These results are also consistent with work on non-human primates finding single-cell activation to both valence and extremity in the amygdala (Paton, Belova, Morrison, \& Salzman, 2006). Interesting, although it was not explicitly examined, it appears that the superficial subnucleus of the amygdala is also the region that showed flexibility in Cunningham et al. (2008). However, as we do not have sufficient spatiotemporal resolution to incisively interrogate amygdala subregions, this conclusion remains highly tentative. Additionally, although these data appear to support the view of a single subnucleus performing multiple functions, previous research has found dissociation within the amygdala between curvilinear (e.g., extremity) and linear (e.g., valence) effects (e.g., Mende-Siedlecki, Said, \& Todorov, 2013). Future research should investigate this potential inconsistency using better localization methods to properly identify which subregions of the amygdala are reacting to which stimuli.

\section{DISCUSSION}

Previous research has advanced two views of the role of the amygdala in processing affective information, characterizing the amygdala as either a "fear center," responding preferentially to negative stimuli (Öhman \& Mineka, 2001; Isenberg et al., 1999; Morris et al., 1996), or alternatively as responsive to relevant information (Whalen, 1998) that may be motivationally or contextually dependent (Cunningham \& Brosch, 2012; Cunningham et al., 2008; Sander et al., 2003). The present study suggests that these inconsistencies might reflect a balance between the need to pursue appetitive goals while maintaining a constant vigilance for potential threats. Specifically, we argue that, because negative information is chronically motivationally relevant, the amygdala will respond to negative stimuli regardless of whether these stimuli are relevant to one's active goals. Because positive stimuli, in general, do not share the status as chronically motivationally relevant, the amygdala may only respond to these stimuli when they are relevant to the current task or goal state.

To test this hypothesis, we showed participants two images simultaneously and gave them the task goal of focusing on one image. We found that amygdala activity was elicited by negative images whether or not they were task relevant. Positive images, however, only led to a relative increase in amygdala activation when those images were task relevant. Together, these data provide initial evidence that the way in which the amygdala responds to task-relevant or task-irrelevant stimuli may differ between classes of stimuli. These results are consistent with the notion that the amygdala responds to motivationally relevant stimuli, such that certain stimuliincluding those linked to negative information-are chronically relevant whereas other stimuli are only relevant in certain contexts.

Taken together, these results are consistent with the view that the amygdala responds preferentially to motivationally relevant information. The current data join a wealth of behavioral research suggesting that what is motivationally relevant is jointly determined by goals that are activated in the specific context as well as those that are chronically relevant to the individual or species. This may allow the amygdala to strike a balance between the need to monitor potential threats as well as flexibly respond to more appetitive goal-relevant stimuli. In fact, the current results complement existing behavioral research demonstrating that, while people do automatically attend to negative stimuli, given the proper ability and motivation, they can show the same sensitivity to positive stimuli (e.g., Smith et al., 2006).

In the present investigation, we have discussed negative stimuli as a class of stimuli that may be chronically motivationally relevant. Negative stimuli, however, need not be the only class of stimuli for which this is the case. For example, previous research has shown that people with anxiety disorders or those who are more neurotic are more likely to have heightened amygdala responses to negative stimuli (Haas, Omura, Constable, \& Canli, 2007; see also Kim \& Whalen, 2009), whereas extraverts and people with greater gain-related motivational styles have a tuning toward positive stimuli when making 
conscious evaluations (Canli, Sivers, Whitfield, Gotlib, \& Gabrieli, 2002). Furthermore, some special classes of stimuli may exist. For example, behavioral evidence suggests that faces may be more chronically relevant for humans than other categories of stimuli (Lavie, Ro, \& Russell, 2003). Indeed, decoding the trustworthiness of a face-whether trustworthy or untrustworthy-is crucially important for social interaction. Across many studies, researchers have found amygdala activation in response to faces regardless of facial valence or the degree to which faces were relevant for the task at hand (e.g., Freeman, Stolier, Ingbretsen, \& Hehman, 2014; Juruena et al., 2010; Said, Dotsch, \& Todorov, 2010). For instance, Todorov, Said, Oosterhof, and Engell (2011) found that the amygdala reliably responded to highly trustworthy and untrustworthy faces regardless of the nature of the task. Recent meta-analyses have similarly found robust activation of the amygdala in response to faces (Engell \& McCarthy, 2013; Mende-Siedlecki, Verosky, Turk-Browne, \& Todorov, 2013; Rossion, Hanseeuw, \& Dricot, 2012; see also Santos, Mier, Kirsch, \& MeyerLindenberg, 2011). Facial stimuli may thus represent a second class of stimuli that is chronically relevant and may therefore elicit an amygdala response regardless of whether the faces are task or goal relevant (Lavie et al., 2003; Todorov et al., 2011; Juruena et al., 2010; but see Wang, Tsuchiya, New, Hurlemann, \& Adolphs, 2014).

\section{Conclusion}

Navigating the world requires attending to and processing important and relevant information in the environment while ignoring objects and details that are less relevant. A functional affective system must balance the complex nature of goal pursuit with the need to avoid fatal errors. In the current study, we propose that the amygdala might help balance the competing motivations of safety with appetitive goal pursuit. Thus, the same brain region that allows us to identify opportunities for a long, cold drink can also help maintain a constant vigilance for predators. This may allow humans and other animals to navigate the environment in a way that will maximize goal pursuit while minimizing catastrophic mistakes.

\section{APPENDIX: LIST OF IAPS IMAGES USED}

Images used: 1019, 1040, 1050, 1051, 1052, 1070, 1090, $1101,1110,1111,1113,1114,1120,1121,1200,1201$, $1205,1220,1230,1270,1274,1275,1280,1300,1301$, $1313,1340,1440,1460,1463,1500,1525,1540,1590$, $1600,1603,1604,1610,1620,1670,1710,1721,1740$, 1750, 1811, 1920, 1930, 1931, 1932, 1947, 1999, 2040, 2050, 2057, 2058, 2070, 2071, 2080, 2091, 2100, 2110, 2120, 2130, 2150, 2160, 2165, 2170, 2190, 2191, 2206, 2208, 2209, 2214, 2216, 2222, 2224, 2235, 2260, 2278,
2299, 2304, 2311, 2312, 2331, 2340, 2341, 2345, 2352.1, 2357, 2360, 2370, 2372, 2381, 2383, 2387, 2388, 2391, 2393, 2395, 2399, 2487, 2490, 2491, 2495, 2499, 2514, 2516, 2518, 2520, 2530, 2540, 2550, 2575, 2579, 2580, 2590, 2595, 2635, 2650, 2661, 2681, 2682, 2692, 2694, 2695, 2700, 2702, 2715, 2722, 2745.1, 2745.2, 2750, 2752, 2753, 2795, 2840, 2850, 2870, 2880, 2890, 2980, 3022, 3250, 3280, 4571, 4599, 4603, 4605, 4610, 4614, 4621, 4622, 4623, 4626, 4640, 4641, 5001, 5010, 5200, $5260,5270,5390,5395,5455,5460,5470,5480,5500$, $5510,5520,5530,5531,5532,5533,5534,5535,5551$, 5594, 5600, 5621, 5623, 5660, 5661, 5700, 5731, 5740, 5760, 5779, 5780, 5811, 5830, 5831, 5891, 5910, 5920, 5971, 5972, 5982, 6000, 6010, 6020, 6150, 6190, 6200, 6210, 6211, 6241, 6244, 6314, 6410, 6555, 6561, 6570.2, 6610, 6800, 6836, 6840, 6940, 7000, 7002, 7004, 7006, 7009, 7010, 7020, 7025, 7030, 7031, 7034, 7035, 7036, $7037,7038,7040,7041,7050,7060,7080,7090,7095$, $7096,7100,7110,7130,7140,7150,7160,7161,7170$, $7175,7179,7180,7182,7183,7185,7186,7187,7190$, $7195,7200,7205,7207,7211,7217,7230,7233,7235$, $7236,7237,7260,7270,7280,7283,7285,7330,7360$, 7430, 7490, 7491, 7495, 7496, 7500, 7502, 7504, 7550, 7580, 7590, 7640, 7710, 7820, 7830, 7950, 8030, 8080, $8160,8170,8180,8185,8190,8200,8210,8211,8231$, $8311,8350,8370,8380,8400,8420,8461,8465,8470$, $8480,8490,8496,8497,8501,8502,8540,9001,9008$, 9010, 9046, 9080, 9090, 9101, 9102, 9110, 9120, 9160, 9171, 9182, 9190, 9230, 9270, 9320, 9341, 9342, 9360, 9373, 9390, 9404, 9409, 9417, 9440, 9470, 9471, 9472, 9480, 9490, 9495, 9584, 9592, 9594, 9621, 9830, 9912

\section{Acknowledgments}

The authors thank Sharon David for help in conducting the study and Norman Farb, the New York University Social Perception and Evaluation Lab, and the University of Toronto Computational Affective Neuroscience Lab for their thoughtful comments on an earlier draft of this manuscript. This research was supported by an NSF grant (BCS-1122352) to William Cunningham. This research was partially supported by a SSHRC CGS to Jay Van Bavel and an NSF graduate research fellowship to Paul Stillman.

Reprint requests should be sent to William A. Cunningham, Department of Psychology, University of Toronto, 100 St. George St., Toronto, Ontario M5S 3G3, Canada, or via e-mail: Cunningham@ psych.utoronto.ca.

\section{Notes}

1. As one reviewer noted, a potential limitation of this study is the unintentional inclusion of primarily female participants.

2. Randomization was such that participants never saw the same image more than twice (once on the left and once on the right).

3. These data were not the focus of this study and are not discussed further.

4. There is, however, an alternative model using trial as the Level 1 variable and hemisphere of activation as the Level 2 variable (keeping subject as the Level 3 variable). If we run this 
alternative model, our results are virtually identical. The reported model is equivalent (when controlling for hemisphere of activation) to averaging the hemisphere of activation together and running a two-level model with trial as the Level 1 variable and subject as the Level 2 variable.

5. This does not, however, rule out the possibility that participants were just following directions at the evaluation task but not the attending task, a point we return to in Discussion.

6. If we divide the data up and run a separate model for both left and right amygdala, we get consistent results, although the $V_{\text {I }}$ coefficient for the left amygdala did not reach conventional levels of significance for the task-irrelevant valence effect $\left(V_{\mathrm{R}}{ }^{2}\right.$; right amygdala: $\gamma=19.75, S E=9.31, p=.03$; left amygdala: $\gamma=$ $28.18, S E=9.51, p=.003 ; V_{\mathrm{I}}$ : right amygdala: $\gamma=-16.00, S E=$ $7.37, p=.03$; left amygdala: $\gamma=-11.94, S E=7.53, p=.11$ ). This is, however, consistent with recent meta-analyses showing that the right amygdala is more responsive to unconsciously perceived stimuli than the left amygdala (Costafreda, Brammer, David, \& Fu, 2008).

\section{REFERENCES}

Adolphs, R., Tranel, D., \& Damasio, A. R. (1998). The human amygdala in social judgment. Nature, 393, 470-474.

Anderson, A. K. (2005). Affective influences on the attentional dynamics supporting awareness. Journal of Experimental Psychology: General, 134, 258-281.

Anderson, A. K., Christoff, K., Panitz, D., De Rosa, E., \& Gabrieli, J. D. (2003). Neural correlates of the automatic processing of threat facial signals. The Journal of Neuroscience, 23, 5627-5633.

Anderson, A. K., Christoff, K., Stappen, I., Panitz, D., Ghahremani, D. G., Glover, G., et al. (2003). Dissociated neural representations of intensity and valence in human olfaction. Nature Neuroscience, 6, 196-202.

Anderson, A. K., \& Phelps, E. A. (2001). Lesions of the human amygdala impair enhanced perception of emotionally salient events. Nature, 411, 305-309.

Bach, D. R., Behrens, T. E., Garrido, L., Weiskopf, N., \& Dolan, R. J. (2011). Deep and superficial amygdala nuclei projections revealed in vivo by probabilistic tractography. The Journal of Neuroscience, 31, 618-623.

Baumeister, R. F., Bratslavsky, E., Finkenauer, C., \& Vohs, K. D. (2001). Bad is stronger than good. Review of General Psychology, 5, 323-370.

Bishop, S. J., Jenkins, R., \& Lawrence, A. D. (2007). Neural processing of fearful faces: Effects of anxiety are gated by perceptual capacity limitations. Cerebral Cortex, 17, $1595-1603$.

Breiter, H. C., Etcoff, N. L., Whalen, P. J., Kennedy, W. A., Rauch, S. L., Buckner, R. L., et al. (1996). Response and habituation of the human amygdala during visual processing of facial expression. Neuron, 17, 875-887.

Brickman, P., Coates, D., \& Janoff-Bulman, R. (1978). Lottery winners and accident victims: Is happiness relative? Journal of Personality and Social Psychology, 36, 917-927.

Bruner, J. S., \& Goodman, C. C. (1947). Value and need as organizing factors in perception. The Journal of Abnormal and Social Psychology, 42, 33.

Canli, T., Sivers, H., Whitfield, S. L., Gotlib, I. H., \& Gabrieli, J. D. (2002). Amygdala response to happy faces as a function of extraversion. Science, 296, 2191.

Canli, T., Zhao, Z., Brewer, J., Gabrieli, J. D., \& Cahill, L. (2000). Event-related activation in the human amygdala associates with later memory for individual emotional experience. Journal of Neuroscience, 20, RC99-1.
Costafreda, S. G., Brammer, M. J., David, A. S., \& Fu, C. H. (2008). Predictors of amygdala activation during the processing of emotional stimuli: A meta-analysis of 385 PET and fMRI studies. Brain Research Reviews, 58, $57-70$.

Cunningham, W. A., \& Brosch, T. (2012). Motivational salience amygdala tuning from traits, needs, values, and goals. Current Directions in Psychological Science, 21, 54-59.

Cunningham, W. A., Johnson, M. K., Gatenby, J. C., Gore, J. C., \& Banaji, M. R. (2003). Neural components of social evaluation. Journal of Personality and Social Psychology, 85, 639-649.

Cunningham, W. A., Raye, C. L., \& Johnson, M. K. (2004). Implicit and explicit evaluation: fMRI correlates of valence, emotional intensity, and control in the processing of attitudes. Journal of Cognitive Neuroscience, 16, 1717-1729.

Cunningham, W. A., Van Bavel, J. J., \& Johnsen, I. R. (2008). Affective flexibility evaluative processing goals shape amygdala activity. Psychological Science, 19, 152-160.

David, J. P., Green, P. J., Martin, R., \& Suls, J. (1997). Differential roles of neuroticism, extraversion, and event desirability for mood in daily life: An integrative model of top-down and bottom-up influences. Journal of Personality and Social Psychology, 73, 149-159.

Davis, M., \& Whalen, P. J. (2001). The amygdala: Vigilance and emotion. Molecular Psychiatry, 6, 13-34.

Engell, A. D., \& McCarthy, G. (2013). Probabilistic atlases for face and biological motion perception: An analysis of their reliability and overlap. Neuroimage, 74, 140-151.

Ferguson, M. J., \& Bargh, J. A. (2004). Liking is for doing: The effects of goal pursuit on automatic evaluation. Journal of Personality and Social Psychology, 87, 557-572.

Förster, J., Liberman, N., \& Friedman, R. S. (2007). Seven principles of goal activation: A systematic approach to distinguishing goal priming from priming of non-goal constructs. Personality and Social Psychology Review, 11, 211-233.

Freeman, J. B., Stolier, R. M., Ingbretsen, Z. A., \& Hehman, E. A. (2014). Amygdala responsivity to high-level social information from unseen faces. The Journal of Neuroscience, 34, 10573-10581.

Goossens, L., Kukolja, J., Onur, O. A., Fink, G. R., Maier, W., Griez, E., et al. (2009). Selective processing of social stimuli in the superficial amygdala. Human Brain Mapping, 30, 3332-3338.

Haas, B. W., Omura, K., Constable, R. T., \& Canli, T. (2007). Emotional conflict and neuroticism: Personality-dependent activation in the amygdala and subgenual anterior cingulate. Behavioral Neuroscience, 121, 249-256.

Hariri, A. R., Tessitore, A., Mattay, V. S., Fera, F., \& Weinberger, D. R. (2002). The amygdala response to emotional stimuli: A comparison of faces and scenes. Neuroimage, 17, 317-323.

Holland, P. C., \& Gallagher, M. (1999). Amygdala circuitry in attentional and representational processes. Trends in Cognitive Sciences, 3, 65-73.

Isenberg, N., Silbersweig, D., Engelien, A., Emmerich, S., Malavade, K., Beattie, B. A., et al. (1999). Linguistic threat activates the human amygdala. Proceedings of the National Academy of Sciences, 96, 10456-10459.

Ito, T. A., Larsen, J. T., Smith, N. K., \& Cacioppo, J. T. (1998). Negative information weighs more heavily on the brain: The negativity bias in evaluative categorizations. Journal of Personality and Social Psychology, 75, 887-900.

Jenkinson, M., Bannister, P., Brady, M., \& Smith, S. (2002). Improved optimisation for the robust and accurate linear registration and motion correction of brain images. Neuroimage, 17, 825-841. 
Jenkinson, M., \& Smith, S. (2001). A global optimisation method for robust affine registration of brain images. Medical Image Analysis, 5, 143-156.

Juruena, M. F., Giampietro, V. P., Smith, S. D., Surguladze, S. A., Dalton, J. A., Benson, P. J., et al. (2010). Amygdala activation to masked happy facial expressions. Journal of the International Neuropsychological Society, 16, 383-387.

Kahneman, D., \& Tversky, A. (1979). Prospect theory: An analysis of decision under risk. Econometrica: Journal of the Econometric Society, 47, 263-291.

Kappenman, E. S., MacNamara, A., \& Proudfit, G. H. (2014). Electrocortical evidence for rapid allocation of attention to threat in the dot-probe task. Social Cognitive and Affective Neuroscience, nsu098.

Kim, M. J., Loucks, R. A., Palmer, A. L., Brown, A. C., Solomon, K. M., Marchante, A. N., et al. (2011). The structural and functional connectivity of the amygdala: From normal emotion to pathological anxiety. Behavioral Brain Research, 223, 403-410.

Kim, M. J., \& Whalen, P. J. (2009). The structural integrity of an amygdala-prefrontal pathway predicts trait anxiety. Journal of Neuroscience, 29, 11614-11618.

Kluver, H., \& Bucy, P. C. (1939). Preliminary analysis of functions of the temporal lobes in monkeys. Archives of Neurology and Psychiatry, 42, 979.

Lang, P. J., Bradley, M. M., \& Cuthbert, B. N. (2008). International affective picture system (IAPS): Affective ratings of pictures and instruction manual. Technical Report A-8, University of Florida, Gainesville, FL.

Lavie, N., Ro, T., \& Russell, C. (2003). The role of perceptual load in processing distractor faces. Psychological Science, 14, $510-515$.

LeDoux, J. E. (2000). Emotion circuits in the brain. Annual Review of Neuroscience, 23, 155-184.

Lindquist, K. A., Wager, T. D., Kober, H., Bliss-Moreau, E., \& Barrett, L. F. (2012). The brain basis of emotion: A metaanalytic review. Behavioral and Brain Sciences, 35, 121-143.

Mende-Siedlecki, P., Said, C. P., \& Todorov, A. (2013). The social evaluation of faces: A meta-analysis of functional neuroimaging studies. Social Cognitive and Affective Neuroscience, 8, 285-299.

Mende-Siedlecki, P., Verosky, S. C., Turk-Browne, N. B., \& Todorov, A. (2013). Robust selectivity for faces in the human amygdala in the absence of expressions. Journal of Cognitive Neuroscience, 25, 2086-2106.

Morris, J. S., Frith, C. D., Perrett, D. I., Rowland, D., Young, A. W., Calder, A. J., et al. (1996). A differential neural response in the human amygdala to fearful and happy facial expressions. Nature, 383, 812-815.

Mumford, J. A., Turner, B. O., Ashby, F. G., \& Poldrack, R. A. (2012). Deconvolving BOLD activation in event-related designs for multivoxel pattern classification analyses. Neuroimage, 59, 2636-2643.

Öhman, A., Flykt, A., \& Esteves, F. (2001). Emotion drives attention: Detecting the snake in the grass. Journal of Experimental Psychology: General, 130, 466-478.

Öhman, A., \& Mineka, S. (2001). Fears, phobias, and preparedness: Toward an evolved module of fear and fear learning. Psychological Review, 108, 483-522.

Paton, J. J., Belova, M. A., Morrison, S. E., \& Salzman, C. D. (2006). The primate amygdala represents the positive and negative value of visual stimuli during learning. Nature, 439, 865-870.

Pessoa, L. (2005). To what extent are emotional visual stimuli processed without attention and awareness? Current Opinion in Neurobiology, 15, 188-196.

Pessoa, L. (2008). On the relationship between emotion and cognition. Nature Reviews Neuroscience, 9, 148-158.
Pessoa, L., McKenna, M., Gutierrez, E., \& Ungerleider, L. G. (2002). Neural processing of emotional faces requires attention. Proceedings of the National Academy of Sciences, 99, 11458-11463.

Pratto, F., \& John, O. P. (1991). Automatic vigilance: The attention-grabbing power of negative social information. Journal of Personality and Social Psychology, 61, 380-391.

Radel, R., \& Clément-Guillotin, C. (2012). Evidence of motivational influences in early visual perception hunger modulates conscious access. Psychological Science, 23, 232-234.

Rossion, B., Hanseeuw, B., \& Dricot, L. (2012). Defining face perception areas in the human brain: A large-scale factorial fMRI face localizer analysis. Brain and Cognition, 79, 138-157.

Rozin, P., \& Royzman, E. B. (2001). Negativity bias, negativity dominance, and contagion. Personality and Social Psychology Review, 5, 296-320.

Said, C. P., Dotsch, R., \& Todorov, A. (2010). The amygdala and FFA track both social and non-social face dimensions. Neuropsychologia, 48, 3596-3605.

Sander, D., Grafman, J., \& Zalla, T. (2003). The human amygdala: An evolved system for relevance detection. Reviews in the Neurosciences, 14, 303-316.

Santos, A., Mier, D., Kirsch, P., \& Meyer-Lindenberg, A. (2011). Evidence for a general face salience signal in human amygdala. Neuroimage, 54, 3111-3116.

Sheldon, K. M., Ryan, R., \& Reis, H. T. (1996). What makes for a good day? Competence and autonomy in the day and in the person. Personality and Social Psychology Bulletin, 22, 1270-1279.

Small, D. M., Gregory, M. D., Mak, Y. E., Gitelman, D., Mesulam, M., \& Parrish, T. (2003). Dissociation of neural representation of intensity and affective valuation in human gustation. Neuron, 39, 701-711.

Smith, N. K., Larsen, J. T., Chartrand, T. L., Cacioppo, J. T., Katafiasz, H. A., \& Moran, K. E. (2006). Being bad isn't always good: Affective context moderates the attention bias toward negative information. Journal of Personality and Social Psychology, 90, 210-220.

Smith, S. (2002). Fast robust automated brain extraction. Human Brain Mapping, 17, 143-155.

Todorov, A., Said, C. P., Oosterhof, N. N., \& Engell, A. D. (2011). Task-invariant brain responses to the social value of faces. Journal of Cognitive Neuroscience, 23, 2766-2781.

Vuilleumier, P. (2005). How brains beware: Neural mechanisms of emotional attention. Trends in Cognitive Sciences, 9, 585-594.

Vuilleumier, P., Armony, J. L., Driver, J., \& Dolan, R. J. (2001). Effects of attention and emotion on face processing in the human brain: An event-related fMRI study. Neuron, 30, 829-841.

Vuilleumier, P., \& Huang, Y. M. (2009). Emotional attention uncovering the mechanisms of affective biases in perception. Current Directions in Psychological Science, 18, 148-152.

Wang, S., Tsuchiya, N., New, J., Hurlemann, R., \& Adolphs, R. (2014). Preferential attention to animals and people is independent of the amygdala. Social Cognitive and Affective Neuroscience, nsu065.

Whalen, P. J. (1998). Fear, vigilance, and ambiguity: Initial neuroimaging studies of the human amygdala. Current Directions in Psychological Science, 7, 177-188.

Whalen, P. J., \& Phelps, E. A. (Eds.) (2009). The buman amygdala. New York: Guilford Press.

Williams, M. A., McGlone, F., Abbott, D. F., \& Mattingley, J. B. (2005). Differential amygdala responses to happy and fearful facial expressions depend on selective attention.

Neuroimage, 24, 417-425. 